Chinese Journal of Organic Chemistry

\title{
6-芳基茚并异喹啉酮衍生物的合成新方法
}

\author{
牛绍雄 ${ }^{a}$ 唐智超 ${ }^{a}$ 刘 畅 ${ }^{c}$ 王天麟 ${ }^{a}$ \\ 尤启冬 $b$ 向 华*,a,b \\ $\left({ }^{a}\right.$ 中国药科大学药学院 江苏省药物分子设计与成药性优化重点实验室＼cjkstart南京 210009) \\ $\left({ }^{b}\right.$ 中国药科大学药学院药物化学教研室 南京 210009)

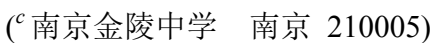

\begin{abstract}
摘要 设计了 6-芳基狮并异喹啉酮衍生物的合成新方法. 该方法运用立体选择性反应策略，专一性地得到 cis-中间体: $c i s-N$-(4-羟基苯基)-1-氧代-3-苯基-1,2,3,4-四氢异喹啉-4-羧酸; 选择以 $\mathrm{KAl}\left(\mathrm{SO}_{4}\right)_{2} \cdot 12 \mathrm{H}_{2} \mathrm{O}$ 为催化剂, 高唒酸、对氨基苯 酚、苯甲醛三组分一锅法反应得到 6-芳基狮并异哇啉酮母核，最终以总收率 36\% 51\%得到 6-芳基狮并异喯啉酮衍生 物, 比原方法的平均收率提高 $15 \%$ 左右, 新方法具有立体选择性好、操作简化、收率高等优点, 适宜大量制备.

关键词 6-芳基苑并异喹啉酮衍生物; 立体选择性反应; cis-中间体; 三组分一锅法; 合成
\end{abstract}

\section{An Improved Synthesis of 6-Arylindenoisoquinoline Derivatives}

\author{
Niu, Shaoxiong ${ }^{a} \quad$ Tang, Zhichao $^{a} \quad$ Liu, Chang $^{c} \quad$ Wang, Tianlin $^{a}$ \\ You, Qidong ${ }^{b} \quad$ Xiang, Hua ${ }^{*, a, b}$ \\ $\left({ }^{a}\right.$ Jiangsu Key Laboratory of Drug Design and Optimization, School of Pharmacy, China Pharmaceutical University, \\ Nanjing 210009) \\ $\left({ }^{b}\right.$ Department of Medicinal Chemistry, School of Pharmacy, China Pharmaceutical University, Nanjing 210009) \\ ( ${ }^{c}$ Jinling High School, Nanjing 210005)
}

\begin{abstract}
An improved process for the synthesis of 6-arylindenoisoquinoline derivatives was developed, and the key intermediate $c i s-N$-(4-hydroxyphenyl)-1-oxo-3-phemyl-1,2,3,4-tetrahydroisoquinoline-4-carboxylic acid was stereoselectively synthesized. The intermediate with 6-arylindenoisoquinoline core could be obtained by an one-pot three-component condensation catalyzed by $\mathrm{KAl}\left(\mathrm{SO}_{4}\right)_{2} \bullet 12 \mathrm{H}_{2} \mathrm{O}$. The overall yield of the target compounds was $36 \% \sim 51 \%$. The improved synthesis method is more suitable for scale-up production with satisfactory stereoselectivity, easily purified procedures and higher yields.
\end{abstract}

Keywords 6-arylindenoisoquinolines; cis-intermediate; stereoselective reaction; one-pot three-component method; synthesis

6-芳基狮并异喹啉酮类衍生物(图 1)具有多种抗肿 瘤作用机制，不仅表现出良好的拓朴异构酶 I (topoisomerase I, Top1)的抑制活性 ${ }^{[1,2]}$, 还能下调䧳激素 受体 $\alpha$ (estrogen receptor $\alpha, \mathrm{ER} \alpha$ ) 和血管内皮生长因子受 体(vascular endothelial growth factor, VEGFR)的表达, 对 $\mathrm{ER}(+)$ 人乳腺癌细胞 $(\mathrm{MCF}-7) 、 \mathrm{ER}(+)$ 人子宫内膜癌 细胞(Ishikawa)、人脐静脉血管内皮细胞(HUVEC)、人
非小细胞肺癌细胞(H460)、人神经胶质瘤细胞(U251)等 表现出较强的抑制活性 ${ }^{[3,4]}$. 其关键中间体四氢异喹啉 羧酸 $\mathbf{2 a}$ (图 1) 也是合成具有多种抗肿瘤活性的天然产物 分子如氯化两面针碱、紫堇灵等的起始原料, 在天然产 物的全合成以及杂环类化合物分子构建中具有广泛的 应用 ${ }^{[5,6]}$.

\footnotetext{
* E-mail: 1020030692@cpu.edu.cn
}

Received April 3, 2015; revised April 27, 2015; published online June 6, 2015.

Project supported by the National Natural Science Foundation of China (No. 81373279), the Twelfth Five-Year Plan Major Project of Candidate Drugs, Ministry of Science and Technology (No. 2012ZX09103101048), the Jiangsu Province Science and Technology Support Program of Social Development Project (No. BE2012745) and the Graduate Innovation Project of Jiangsu Province (No. KYLX 0612).

国家自然科学基金(No. 81373279)、科技部 “十二五” 新药创制重大专项(No. 2012ZX09103101048)、江苏省科技支撑计划(No. BE2012745)和江苏省 研究生科研创新计划(No. KYLX_0612)资助项目. 

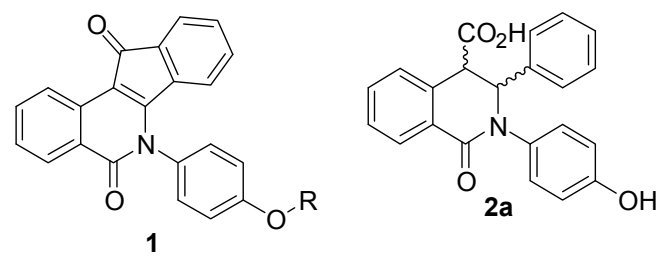

图 1 6-芳基狮并异喹啉酮(1)与中间体四氢异喹啉酮羧酸 (2a) Figure 1 6-Arylindenoisoquinoline (1) and intermediate tetrahydroisoquinoline acid (2a)

6-芳基狮并异喹啉酮类衍生物的合成最初采用 Cushman 报道的两步合成法(Scheme 1) ${ }^{[3,7]}$, 即先由对氨 基苯酚(3)和苯甲醛(4a)制备 4-芐烯氨基苯酚(5), 再通过 5 和高酞酸酐 $(6 a)$ 在二氯甲烷中发生缩合反应, 得到中 间体四氢异喹啉羧酸(2a)的 cis-和 trans-异构体的混合 物; 其中 cis-2a 依次在二氯亚砜和三氯化铝的作用下发 生脱氢、环合生成 6-芳基䒢并异喹啉酮母核 7, 最终经 过氧烃基化反应接上含氮侧链从而完成 6-芳基狮并异 喹啉酮衍生物的合成. 由于反应生成的 trans-2a 需要酯 化后依次在正丁基锂和甲基磺酰氯的作用下才能发生 脱氢反应, 且 trans-2a 无法通过反应转化为 $c i s-\mathbf{2} \mathbf{a}^{[7,8]}$, 因此成为合成过程中的杂质, 增大了分离的难度, 也减 少了目标化合物的生成量, 造成终产物总收率仅为 $17 \% \sim 33 \%{ }^{[3,8]}$.
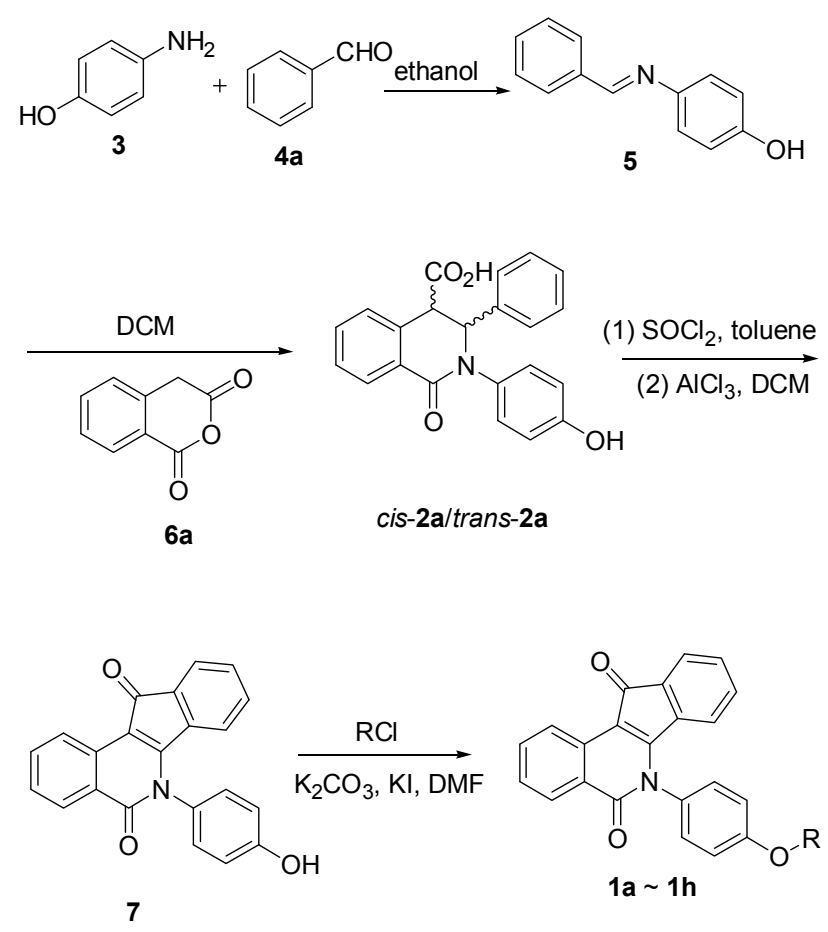

图式 1 6-芳基䒢并异喹啉酮(1)的一般合成方法

Scheme 1 General preparation of 6-arylindenoisoquinoline (1)

由于 $c i s-2 \mathbf{a}$ 产率的高低是决定目标产物收率的关

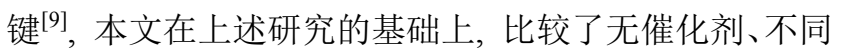
的质子酸或路易斯酸做催化剂时, 5 和 $6 \mathrm{a}$ 经缩合反应生
成 cis-2a 的收率; 并进一步探索了三组分一锅法替代原 来的二步法合成 cis-2a 的反应条件, 优选出了合成 6-芳 基狮并异喹啉酮类衍生物的新路线(Scheme 2). 此改进 的合成新方法具有立体选择性好、操作简化、收率高等 优点，适宜 6-芳基狮并异喹啉酮类衍生物的大量制备.

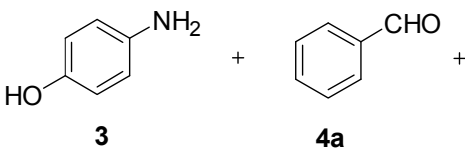

3

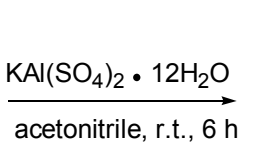<smiles>O=C1Cc2ccccc2C(=O)O1</smiles>

$6 a$

$$
\overrightarrow{\text { acetonitrile, r.t., } 6 \mathrm{~h}}
$$<smiles>O=C(O)[C@H]1c2ccccc2C(=O)N(c2ccc(O)cc2)[C@H]1c1ccccc1</smiles>

cis-2a

图式 2 三组分一锅法选择性合成 $c i s-\mathbf{2 a}$

Scheme 2 Stereoselective synthesis of cis-2a by one-pot threecomponent reaction

\section{1 结果与讨论}

\section{1 中间体 $2 \mathrm{a}$ 的结构确证}

本文合成中所涉及的中间体 $\mathbf{2 a}$ 的构型通过其 ${ }^{1} \mathrm{H}$ NMR 中 3-H 和 4-H 之间的偶合常数大小确定：cis-异构 体的 3-H 和 4-H 处于环的同侧，两 $\mathrm{C}-\mathrm{H}$ 轨道交盖最大， 偶合作用强，邻偶偶合常数 ${ }^{3} J$ 一般在 $5 \sim 8 \mathrm{~Hz}$ 之间, ${ }^{1} \mathrm{H}$ NMR 图谱中 3-H 和 4-H 各自裂分为 $\mathrm{d}$ 峰; trans-异构体 的 3-H 和 4-H 处于环的异侧, 两 $\mathrm{C}-\mathrm{H}$ 轨道二面角趋于 $90^{\circ}$, 相互交盖最小, 偶合作用很弱, 邻偶偶合常数 ${ }^{3} J$ 一 般在 $0 \sim 2 \mathrm{~Hz}$ 之间, ${ }^{1} \mathrm{H}$ NMR 图谱中 3-H 和 4-H 一般表 现为 $\mathrm{s}$ 峰. 对中间体 2a 进行 ${ }^{1} \mathrm{H}$ NMR 检测后可以看出, 其 3-H 和 4-H 各自裂分为 $\mathrm{d}$ 峰, 且偶合常数 ${ }^{3} J=5.7 \mathrm{~Hz}$, 符合 $c i s$-异构体的特征, 并且和相关文献中所报道的结 构特征一致 ${ }^{[5,6]}$, 可以确证中间体 2a 为 cis-异构体(图 2).

\section{2 两步法无催化剂条件下 5 和 $6 a$ 缩合时反应条件篮 选}

首先考察了两步法合成中，无催化剂条件下不同溶 剂和反应温度对第二步亚胺和高酞酸酐缩合的影响. 以 4-芐烯氨基苯酚 5 和高酞酸䣶 $6 \mathbf{a}$ 为原料, 在非催化条件 下，考察 3 种常用且易于后处理的溶剂和不同的反应温 度对 cis-2a 收率的影响(表 1). 反应监测结束后后处理纯 化, 单步收率以分离得到的 cis-2a 纯品计算. 结果表明, 该缩合反应受溶剂、反应温度等条件影响较大. 尽管以 乙腈为溶剂, $80{ }^{\circ} \mathrm{C}$ 下回流反应可以得到 55\%的收率，但 是两步法中第一步对氨基苯酚 3 和苯甲醛 $4 \mathrm{a}$ 缩合生成 5 的收率为 $87 \%$, 故无催化剂条件下两步法的最高收率仅 


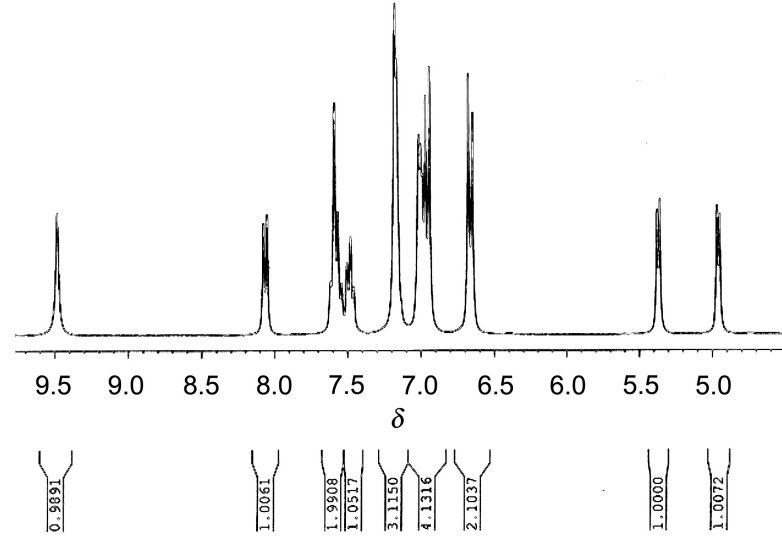

图 2 中间体 $2 \mathbf{a}$ 的部分 ${ }^{1} \mathrm{H}$ NMR 图谱

Figure 2 Partial ${ }^{1} \mathrm{H}$ NMR spectrum of $\mathbf{2 a}$

为 $48 \%$, 其结果较原方法虽有提高, 但仍不理想.

表 1 反应条件对两步法无催化剂条件下 5 和 $6 \mathrm{a}$ 缩合反应的 影响 $^{a}$

Table 1 Effect of different reaction conditions on the condensation of $\mathbf{5}$ and $\mathbf{6 a}$ in non-catalytic system

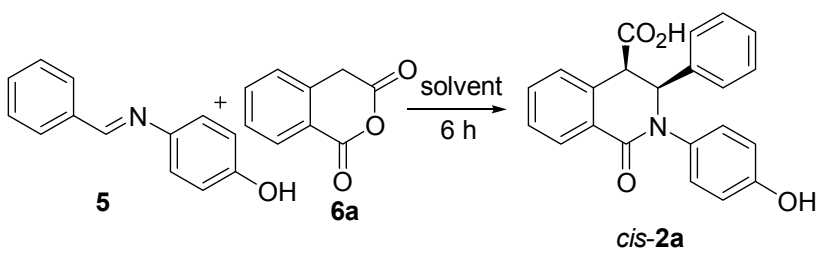

\begin{tabular}{clcc}
\hline Entry & Solvent & Temp. $/{ }^{\circ} \mathrm{C}$ & Yield $^{b} / \%$ \\
\hline 1 & Acetonitrile & r.t & 41 \\
2 & THF & r.t & 35 \\
3 & Chloroform & r.t & 40 \\
4 & Acetonitrile & Reflux & 55 \\
5 & THF & Reflux & 39 \\
6 & Chloroform & Reflux & 43 \\
\hline
\end{tabular}

反应条件: 5 (30.9 mmol), 6a (30.9 mmol, 1 equv.), 溶剂 $60 \mathrm{~mL} ;{ }^{b}$ 反应收率 均为分离产率.

\section{3 质子酸或路易斯酸催化下 5 和 $6 a$ 缩合时反应条件 筛选}

考虑到非催化体系中 cis-2a 的收率低, 而且重现性 差，本文将立体选择性合成策略引入到 6-芳基狮并异喹 啉酮衍生物的合成工作中. 根据文献报道, 质子酸和路 易斯酸在选择性合成 cis-/trans-四氢异喹啉羧酸类化合 物中有着广泛的应用, 如 silica sulfuric acid、 $\mathrm{KAl}\left(\mathrm{SO}_{4}\right)_{2}$ • $12 \mathrm{H}_{2} \mathrm{O} 、 \mathrm{Yb}(\mathrm{OTf})_{3}$ 或 $\mathrm{I}_{2}$ 等作为催化剂能选择性地得到 $c i s-$ 异构体 ${ }^{[10]}$, 而 $\mathrm{TiCl}_{4}$ 或 $\mathrm{BF}_{3} \cdot \mathrm{Et}_{2} \mathrm{O}$ 催化则主要得到 trans异构体 ${ }^{[11,12]}$. 本文比较了以 $\mathrm{I}_{2}$ 、 silica sulfuric acid、 $\mathrm{KAl}\left(\mathrm{SO}_{4}\right)_{2} \cdot 12 \mathrm{H}_{2} \mathrm{O}$ 或 $\mathrm{Yb}(\mathrm{OTf})_{3}$ 作为催化剂, 在相同的投 料量、溶剂和反应温度条件下, 对二步合成法由 5 和 $6 \mathbf{a}$ 缩合得到 cis-2a 的影响(表 2). 结果表明, 这些催化剂均
能大大提高 $c i s-2 \mathbf{a}$ 的收率, 以 $\mathrm{KAl}\left(\mathrm{SO}_{4}\right)_{2} \bullet 12 \mathrm{H}_{2} \mathrm{O}$ 效果最 优(表 2, Entry 4), 且 $\mathrm{KAl}\left(\mathrm{SO}_{4}\right)_{2} \bullet 12 \mathrm{H}_{2} \mathrm{O}$ 相对于其他 3 种 催化剂有两大优点: 第一, $\mathrm{KAl}\left(\mathrm{SO}_{4}\right)_{2} \cdot 12 \mathrm{H}_{2} \mathrm{O}$ 易于从反 应体系或者产品中分离除去, 反应液通过抽滤、水洗即 可得到 $c i s-\mathbf{2 a}$ 的纯品, 无需萃取、洗涤或者柱层析分离 纯化; 第二, $\mathrm{KAl}\left(\mathrm{SO}_{4}\right)_{2} \cdot 12 \mathrm{H}_{2} \mathrm{O}$ 相对其他 3 种催化剂最易 获得, 价格也最为低廉, 对环境的危害也最低, 非常适 合大规模的合成制备. 综上，我们最终选择 $\mathrm{KAl}\left(\mathrm{SO}_{4}\right)_{2} \cdot 12 \mathrm{H}_{2} \mathrm{O}$ 作为立体选择性合成 cis-2a 的催化剂. 随后，考察了不同溶剂和反应温度对 $\mathrm{KAl}\left(\mathrm{SO}_{4}\right)_{2} \bullet 12 \mathrm{H}_{2} \mathrm{O}$ 催化效率的影响(表 2, Entries 5 10), 其中以乙腈为溶 剂，室温下反应收率最佳(表 2, Entry 5)，可高达 90\%， 加上两步法中第一步对氨基苯酚和苯甲醛缩合生成 $\mathbf{5}$ 的 收率 $87 \%, \mathrm{KAl}\left(\mathrm{SO}_{4}\right)_{2} \cdot 12 \mathrm{H}_{2} \mathrm{O}$ 催化下两步法的最高收率 高达 $78 \%$.

表 2 不同反应条件对质子酸或 Lewis 酸催化下 5 和 $6 \mathrm{a}$ 缩合 反应的影响 ${ }^{a}$

Table 2 Effect of different reaction conditions on the condensation of $\mathbf{5}$ and $\mathbf{6 a}$ catalyzed by protonic acid or Lewis acid

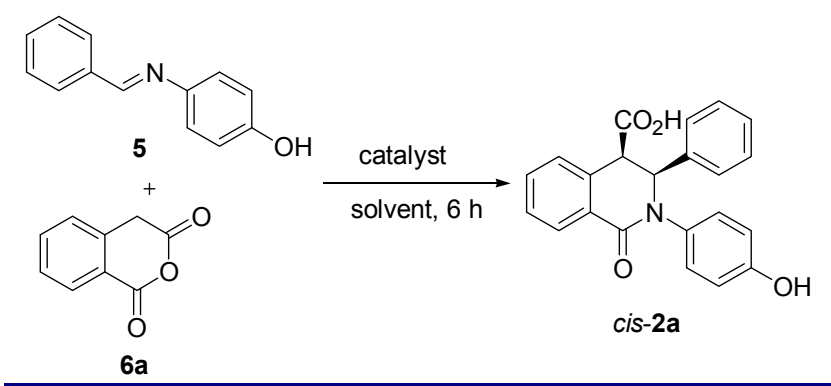

\begin{tabular}{cllcc}
\hline Entry & \multicolumn{1}{c}{ Catalyst } & \multicolumn{1}{c}{ Solvent } & Temp. ${ }^{\circ} \mathrm{C}$ & Yield $^{b} / \%$ \\
\hline 1 & $\mathrm{I}_{2}$ & $\mathrm{CH}_{2} \mathrm{Cl}_{2}$ & r.t & 83 \\
2 & Silica sulfuric acid & $\mathrm{CH}_{2} \mathrm{Cl}_{2}$ & r.t & 80 \\
3 & $\mathrm{Yb}(\mathrm{OTf})_{3}$ & $\mathrm{CH}_{2} \mathrm{Cl}_{2}$ & r.t & 84 \\
4 & $\mathrm{KAl}\left(\mathrm{SO}_{4}\right)_{2} \bullet 12 \mathrm{H}_{2} \mathrm{O}$ & $\mathrm{CH}_{2} \mathrm{Cl}_{2}$ & r.t & 87 \\
5 & $\mathrm{KAl}\left(\mathrm{SO}_{4}\right)_{2} \bullet 12 \mathrm{H}_{2} \mathrm{O}$ & Acetonitrile & r.t & 90 \\
6 & $\mathrm{KAl}\left(\mathrm{SO}_{4}\right)_{2} \cdot 12 \mathrm{H}_{2} \mathrm{O}$ & $\mathrm{THF}$ & r.t & 85 \\
7 & $\mathrm{KAl}\left(\mathrm{SO}_{4}\right)_{2} \bullet 12 \mathrm{H}_{2} \mathrm{O}$ & Chloroform & r.t & 76 \\
8 & $\mathrm{KAl}\left(\mathrm{SO}_{4}\right)_{2} \bullet 12 \mathrm{H}_{2} \mathrm{O}$ & Acetonotrile & 40 & 87 \\
9 & $\mathrm{KAl}\left(\mathrm{SO}_{4}\right)_{2} \bullet 12 \mathrm{H}_{2} \mathrm{O}$ & Acetonotrile & 60 & 70 \\
10 & $\mathrm{KAl}\left(\mathrm{SO}_{4}\right)_{2} \cdot 12 \mathrm{H}_{2} \mathrm{O}$ & Acetonotrile & Reflux & 66 \\
\hline
\end{tabular}

反应条件: 5 (30.9 mmol), 6a (30.9 mmol, 1 equiv.), 催化剂 (15.5 mmol, 0.5 equiv.), 溶剂 $60 \mathrm{~mL} ;^{b}$ 反应收率均为分离产率.

\section{$1.4 \mathrm{KAI}\left(\mathrm{SO}_{4}\right)_{2} \cdot 12 \mathrm{H}_{2} \mathrm{O}$ 催化下 3,4 和 6 三组分一锅法} 反应条件篮选

最后, 设计并实施了 $\mathrm{KAl}\left(\mathrm{SO}_{4}\right)_{2} \bullet 12 \mathrm{H}_{2} \mathrm{O}$ 催化下三组 分一锅法选择性合成 cis-2a 的反应路线, 将两步法简化 为一锅法, 减少反应步骤并提高收率 ${ }^{[13,14]}$. 以 $\mathrm{KAl}\left(\mathrm{SO}_{4}\right)_{2} \cdot 12 \mathrm{H}_{2} \mathrm{O}$ 催化下为优选催化剂, 以乙腈为优选 溶剂, 对氨基苯酚 $(3)$, 苯甲醛 $(4 a)$ 和高酞酸䣶(6a)为原 
料, 无水硫酸镁为缚水剂, 考察了反应温度对 cis-2a 收 率的影响(表 3, Entries 1 4). 其中以室温下反应最佳 (表 3, Entry 1), 可达 $85.5 \%$, 高于 $\mathrm{KAl}\left(\mathrm{SO}_{4}\right)_{2} \bullet 12 \mathrm{H}_{2} \mathrm{O}$ 催化 下两步法收率 78\%，同时也将两步反应缩短为一步. 基 于此策略，目标物 $\mathbf{1 a} \sim \mathbf{1 h}$ 的总收率可从 $17 \% \sim 33 \%$ 提 高至 $36 \% \sim 51 \%$ (表 4). 随后我们在优选反应条件下对 反应底物做了一定的拓展, 以对溴苯甲醛(4b)和 4-甲氧 基高酞酸䣶(6b)作为新反应底物, 在相同反应条件下合 成了 cis-2b 和 $c i s-2 \mathbf{c}($ 表 3, Entries 5 6), 其收率与 $c i s-\mathbf{2 a}$ 没有较大的差距.

表 3 不同反应温度和底物对 $\mathrm{KAl}\left(\mathrm{SO}_{4}\right)_{2} \cdot 12 \mathrm{H}_{2} \mathrm{O}$ 催化下三组分 一锅法合成的影响 ${ }^{a}$

Table 3 Effect of different reaction temperature and substrates on the one-pot three-component method catalyzed by $\mathrm{KAl}\left(\mathrm{SO}_{4}\right)_{2}$ • $12 \mathrm{H}_{2} \mathrm{O}$

\begin{tabular}{cccccc}
\hline Entry & $\mathrm{R}^{1}$ & $\mathrm{R}^{2}$ & Product & Temp. $/{ }^{\circ} \mathrm{C}$ & Yield $^{b} / \%$ \\
\hline 1 & $\mathrm{H}$ & $\mathrm{H}$ & cis-2a & r.t & 86 \\
2 & $\mathrm{H}$ & $\mathrm{H}$ & cis-2a & 40 & 77 \\
3 & $\mathrm{H}$ & $\mathrm{H}$ & cis-2a & 60 & 69 \\
4 & $\mathrm{H}$ & $\mathrm{H}$ & cis-2a & Reflux & 54 \\
5 & $\mathrm{CH}_{3} \mathrm{O}$ & $\mathrm{H}$ & cis-2b & r.t & 88 \\
6 & $\mathrm{CH}_{3} \mathrm{O}$ & $\mathrm{Br}$ & cis-2c & r.t & 85 \\
\hline
\end{tabular}

${ }^{a}$ 反应条件: 3 (30.9 mmol), 4 (30.9 mmol, 1 equiv.), 6 (30.9 mmol, 1 equiv.), $\mathrm{KAl}\left(\mathrm{SO}_{4}\right)_{2} \cdot 12 \mathrm{H}_{2} \mathrm{O}(15.5 \mathrm{mmol}, 0.5$ equiv. $)$, 无水 $\mathrm{MgSO}_{4}(61.8 \mathrm{mmol}, 2$ equiv.), 乙腈 $60 \mathrm{~mL}$, 反应时间 $6 \mathrm{~h} ;^{b}$ 反应收率均为分离产率.

表 4 反应条件改进后目标物 $1 \mathrm{a} \sim 1 \mathrm{~h}$ 的收率

Table 4 Yields of $\mathbf{1 a} \sim \mathbf{1 h}$ by the improved synthesis method

Compd.

\section{2 结论}

通过利用立体选择性合成策略和多组分一锅法，设 计并优化了 6-芳基茚并异喹啉酮类化合物的合成路线, 并对关键反应步骤的条件进行篎选，得到了优化的反应 条件. 采用 $\mathrm{KAl}\left(\mathrm{SO}_{4}\right)_{2} \cdot 12 \mathrm{H}_{2} \mathrm{O}$ 选择性地合成关键中间体 $c i s-2 \mathbf{a}$, 具有立体选择性好、收率高、反应条件温和和操 作简单等优点, 且 $\mathrm{KAl}\left(\mathrm{SO}_{4}\right)_{2} \cdot 12 \mathrm{H}_{2} \mathrm{O}$ 价格低廉, 容易分 离, 对环境危害小, 适于大批量合成和工业化生产; 采 用三组分一锅法替代两步法合成 $c i s-\mathbf{2} \mathbf{a}$, 减少了反应步 骤和物料损失, 间接地提高了 cis-2a 的生成. 通过以上 改进，目标化合物 1a 1h 的总收率由最初的 17\% $33 \%$ 提高至 36\% 51\%(表 4), 成效显著，同时本方法也 为四氢异喹啉类和 6-芳基茚并异喹啉酮类化合物的合 成和衍生化提供了便捷的合成方法.

\section{3 实验部分}

\section{1 仪器与试剂}

化合物的熔点由采用 MELT-TEMP 2 熔点仪测定; IR 用 Nicolet Impact 410 型傅里叶变换红外光谱仪测定 (KBr 压片); 质谱由岛津 GC-MS 2050 型气质联用仪 (EI-MS)或 Agilent 1946A-MSD 型质谱仪(ESI-MS)测定; 氢谱使用 Bruker AV300 型(300 MHz)或 Bruker AV400 型(400 MHz)核磁共振仪测定, DMSO- $d_{6}$ 或 $\mathrm{CDCl}_{3}$ 作溶 剂, TMS 作为内标; 柱层析采用 100 200 目硅胶(青岛 海浪硅胶干燥剂厂). 除特别说明外, 所有溶剂均为市 售分析纯试剂, 干燥的溶剂按照常规的标准方法制得.

\section{2 cis- $N$-(4-羟基苯基)-1-氧代-3-苯基-1,2,3,4-四氢异 喹啉-4-羧酸(cis-2a)的合成实验方法}

先将 $5.01 \mathrm{~g}(30.9 \mathrm{mmol})$ 的高酞酸 $6 \mathbf{a}$ 溶于 $60 \mathrm{~mL}$ 乙

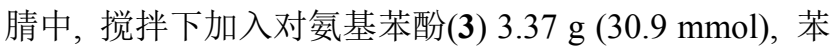
甲醛(4a) $3.14 \mathrm{~mL}(30.9 \mathrm{mmol})$, 随后加入 $\mathrm{KAl}\left(\mathrm{SO}_{4}\right)_{2}$ ・ $12 \mathrm{H}_{2} \mathrm{O} \quad 7.35 \mathrm{~g}$ (15.5 mmol)和无水 $\mathrm{MgSO}_{4} 7.4 \mathrm{~g}(61.8$ $\mathrm{mmol})$, 室温下反应 $8 \mathrm{~h}$, 反应结束后, 减压蒸除大部分 溶剂, 残余物加入 $100 \mathrm{~mL}$ 水化开并搅拌 $10 \mathrm{~min}$, 抽滤, 依次用甲醇 $(25 \mathrm{~mL})$ 和水 $(25 \mathrm{~mL})$ 洗涤滤饼, 干燥后得白 色固体 $9.49 \mathrm{~g}^{[3]}$, 收率 86\%. m.p. 192 193 ${ }^{\circ} \mathrm{C} ;{ }^{1} \mathrm{H}$ NMR $\left(300 \mathrm{MHz}, \mathrm{DMSO}-d_{6}\right) \delta: 9.49(\mathrm{~s}, 1 \mathrm{H}, \mathrm{OH}), 8.06$ (d, $J=7.4$ $\mathrm{Hz}, 1 \mathrm{H}, \mathrm{ArH}), 7.46 \sim 7.62(\mathrm{~m}, 3 \mathrm{H}, \mathrm{ArH}), 7.09 \sim 7.30(\mathrm{~m}$, $3 \mathrm{H}, \mathrm{ArH}), 6.99 \sim 7.06(\mathrm{~m}, 2 \mathrm{H}, \mathrm{ArH}), 6.96(\mathrm{~d}, J=8.6 \mathrm{~Hz}$, $2 \mathrm{H}, \mathrm{ArH}), 6.67(\mathrm{~d}, J=8.6 \mathrm{~Hz}, 2 \mathrm{H}, \mathrm{ArH}), 5.37$ (d, $J=5.7$ $\mathrm{Hz}, 1 \mathrm{H}, 3-\mathrm{CH}), 4.96$ (d, J=5.7 Hz, 1H, 4-CH); MS (ESI) $\mathrm{m} / \mathrm{z}: 360[\mathrm{M}+\mathrm{H}]^{+}$. 
$3.3 \mathrm{cis}-\mathrm{N}$-(4-羟基苯基)-1-氧代-7-甲氧基-3-苯基1,2,3,4-四氢异喹啉-4-羧酸 (cis-2b)的合成

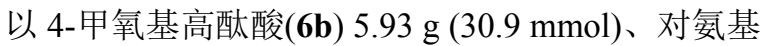
苯酚(3) $3.37 \mathrm{~g}$ (30.9 mmol), 苯甲醛 $(\mathbf{4 a}) 3.14 \mathrm{~mL}$ (30.9 $\mathrm{mmol})$ 为原料, 采用与 cis-2a 相同的合成方法, 得白色 固体 $10.9 \mathrm{~g}$, 收率 $88 \%{ }^{[3]}$. m.p. $162 \sim 164{ }^{\circ} \mathrm{C} ;{ }^{1} \mathrm{H}$ NMR $\left(300 \mathrm{MHz}, \mathrm{DMSO}-d_{6}\right) \delta: 9.49(\mathrm{~s}, 1 \mathrm{H}, \mathrm{OH}), 7.52 \sim 7.57(\mathrm{~m}$, $2 \mathrm{H}, \mathrm{ArH}), 7.11 \sim 7.18(\mathrm{~m}, 4 \mathrm{H}, \mathrm{ArH}), 6.99 \sim 7.09(\mathrm{~m}, 2 \mathrm{H}$, ArH), 6.94 (d, $J=8.6 \mathrm{~Hz}, 2 \mathrm{H}, \mathrm{ArH}), 6.66(\mathrm{~d}, J=8.6 \mathrm{~Hz}$, 2H, ArH), 5.32 (d, $J=5.7 \mathrm{~Hz}, 1 \mathrm{H}, 3-\mathrm{CH}), 4.88$ (d, $J=5.7$ $\mathrm{Hz}, 1 \mathrm{H}, 4-\mathrm{CH}$ ), 3.83 (s, 3H, 7-OCH ${ }_{3}$ ); MS (ESI) $m / z: 390$ $[\mathrm{M}+\mathrm{H}]^{+}$.

3.4 cis- $N$-(4-羟基苯基)-1-氧代-3-(4-溴苯基)-7-甲氧 基-1,2,3,4-四氢异喹啉-4-羧酸(cis-2c)的合成

以 4-甲氧基高酞酸(6b) $5.93 \mathrm{~g}(30.9 \mathrm{mmol})$ 、对氨基 苯酚(3) $3.37 \mathrm{~g}$ (30.9 mmol)、对溴苯甲醛 $(4 \mathrm{~b}) 5.7 \mathrm{~g}$ (30.9 $\mathrm{mmol}$ )为原料, 采用与 cis-2a 相同的合成方法, 得白色 固体 $12.3 \mathrm{~g}^{[3]}$, 收率 85\%. m.p. $143 \sim 146{ }^{\circ} \mathrm{C} ;{ }^{1} \mathrm{H}$ NMR $\left(300 \mathrm{MHz}, \mathrm{DMSO}-d_{6}\right) \delta: 9.51(\mathrm{~s}, 1 \mathrm{H}, \mathrm{OH}), 7.48 \sim 7.59$ (m, 2H, ArH), 7.39 (d, $J=8.3 \mathrm{~Hz}, 2 \mathrm{H}, \mathrm{ArH}), 7.16$ (d, $J=$ $8.3 \mathrm{~Hz}, 2 \mathrm{H}, \mathrm{ArH}), 6.95$ (d, J=7.3 Hz, 4H, ArH), 6.68 (d, $J=8.7 \mathrm{~Hz}, 1 \mathrm{H}, \mathrm{ArH}), 5.34$ (d, $J=5.8 \mathrm{~Hz}, 1 \mathrm{H}, 3-\mathrm{CH}), 4.86$ (d, $J=5.8 \mathrm{~Hz}, 1 \mathrm{H}, 4-\mathrm{CH}), 3.83$ (s, $3 \mathrm{H}, 7-\mathrm{OCH}_{3}$ ); MS (ESI) $m / z: 506[\mathrm{M}+\mathrm{K}]^{+}$.

\subsection{6-(4-羟基苯基)-5H-狮并-[1,2-c]异喹啉-5,11(6H)-} 二酮(7)的合成

取 cis-2a $4.25 \mathrm{~g}(11.8 \mathrm{mmol})$ 于 $100 \mathrm{~mL}$ 无水甲苯中, 搅拌下滴加二氯亚砜 $17.1 \mathrm{~mL}(236 \mathrm{mmol})$, 加毕后升温 回流 $2 \mathrm{~h}$, 反应结束后冷却至室温, 减压蒸除溶剂和二 氯亚砜, 剩余固体用 $100 \mathrm{~mL}$ 无水二氯甲烷溶解, 加入 三氯化铝 $4.72 \mathrm{~g}(35.4 \mathrm{mmol})$, 回流反应 $4 \mathrm{~h}$, 反应结束 后, 冷却至室温, 于冰浴中摚拌并缓缓加入 $100 \mathrm{~mL}$ 水 猝灭反应, 于 $500 \mathrm{~mL}$ 分液漏斗中分液, 油相用 $100 \mathrm{~mL}$ 水洗涤 2 次, 干燥, 减压蒸除溶剂, 所得粗品用 $20 \mathrm{~mL}$ 四氢呋喃重结晶, 得红色粉末状固体 $2.92 \mathrm{~g}$, 收率 $73 \%{ }^{[3]}$. m.p. $278 \sim 280{ }^{\circ} \mathrm{C} ;{ }^{1} \mathrm{H}$ NMR $(300 \mathrm{MHz}$, DMSO- $\left.d_{6}\right) \delta: 10.07(\mathrm{~s}, 1 \mathrm{H}, \mathrm{OH}), 8.75(\mathrm{~d}, J=8.3 \mathrm{~Hz}, 1 \mathrm{H}$, ArH), 8.35 (d, $J=8.3 \mathrm{~Hz}, 1 \mathrm{H}, \operatorname{ArH}), 7.76(\mathrm{t}, J=8.3 \mathrm{~Hz}$, 1H, ArH), 7.55 (d, $J=7.3 \mathrm{~Hz}, 1 \mathrm{H}, \operatorname{ArH}), 7.49$ (t, $J=7.3$ $\mathrm{Hz}, 1 \mathrm{H}, \mathrm{ArH}), 7.36$ (d, $J=8.7 \mathrm{~Hz}, 2 \mathrm{H}, \mathrm{ArH}), 7.25$ (t, $J=$ $7.6 \mathrm{~Hz}, 1 \mathrm{H}, \mathrm{ArH}), 7.15$ (d, $J=8.7 \mathrm{~Hz}, 2 \mathrm{H}, \mathrm{ArH}), 7.04$ (t, $J=7.6 \mathrm{~Hz}, 1 \mathrm{H}, \mathrm{ArH}), 5.64$ (d, $J=7.6 \mathrm{~Hz}, 1 \mathrm{H}, \mathrm{ArH}) ; \mathrm{MS}$ (EI) $m / z: 339[\mathrm{M}]^{+}$.

\subsection{6-芳基狮并异喹啉酮衍生物 1 的合成}

3.6.1 6-[4-(2-哌啶基乙氧基)苯基]- $5 \mathrm{H}$-狮并 $[1,2-c]$ 异 喹啉-5,11(6H)-二酮(1a)的合成

取化合物 $82.71 \mathrm{~g}(8.0 \mathrm{mmol})$ 和 $N$-(2-氯乙基)哌啶盐

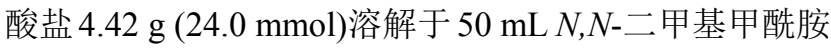

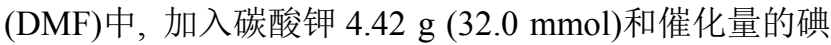
化钾, 氮气保护下 $90{ }^{\circ} \mathrm{C}$ 下回流 $8 \mathrm{~h}$, 反应结束后冷却至 室温, 缓缓加入到 $100 \mathrm{~mL}$ 冷水中, 搅拌, 抽滤, $20 \mathrm{~mL}$ 水洗滤饼, 干燥, 所的粗品用 $V$ (二氯甲烷) $: V$ (甲醇 $)=$ $20: 1$ 柱层析得精品, 深红色粉末状固体 $2.31 \mathrm{~g}^{[3]}$, 收率 64\%. m.p. $98 \sim 102{ }^{\circ} \mathrm{C} ;{ }^{1} \mathrm{H}$ NMR $\left(400 \mathrm{MHz}, \mathrm{CDCl}_{3}\right) \delta$ : $8.74(\mathrm{~d}, J=8.0 \mathrm{~Hz}, 1 \mathrm{H}, \mathrm{ArH}), 8.37(\mathrm{~d}, J=8.0 \mathrm{~Hz}, 1 \mathrm{H}$, ArH), 7.78 (t, $J=8.0 \mathrm{~Hz}, 1 \mathrm{H}, \mathrm{ArH}), 7.57(\mathrm{~d}, J=7.2 \mathrm{~Hz}$, $1 \mathrm{H}, \operatorname{ArH}), 7.50$ (t, $J=7.2 \mathrm{~Hz}, 1 \mathrm{H}, \operatorname{ArH}), 7.35$ (d, $J=8.8$ $\mathrm{Hz}, 2 \mathrm{H}, \mathrm{ArH}), 7.25$ (t, $J=7.6 \mathrm{~Hz}, 1 \mathrm{H}, \mathrm{ArH}), 7.15$ (d, $J=$ $8.8 \mathrm{~Hz}, 2 \mathrm{H}, \mathrm{ArH}), 7.07$ (t, $J=7.6 \mathrm{~Hz}, 1 \mathrm{H}, \mathrm{ArH}), 5.68$ (d, $J=7.6 \mathrm{~Hz}, 1 \mathrm{H}, \mathrm{ArH}), 4.26\left(\mathrm{t}, J=6.0 \mathrm{~Hz}, 2 \mathrm{H}, \mathrm{OCH}_{2-}\right.$ $\mathrm{CH}_{2} \mathrm{~N}$ ), $2.89\left(\mathrm{t}, J=6.0 \mathrm{~Hz}, 2 \mathrm{H}, \mathrm{OCH}_{2} \mathrm{CH}_{2} \mathrm{~N}\right), 2.50 \sim 2.71$ $(\mathrm{m}, \quad 4 \mathrm{H}, \quad 2,6$-piperidyl-H), $1.65 \sim 1.71(\mathrm{~m}, 4 \mathrm{H}, \quad 3,5-$ piperidyl-H), $1.50 \sim 1.52$ (m, 2H, 4-piperidyl-H); IR (KBr) $v: 3545,3421,2925,1669,1607,1503,1412,1247,759$, $530 \mathrm{~cm}^{-1}$; MS (EI) $\mathrm{m} / z: 450[\mathrm{M}]^{+}$.

3.6.2 6-[4-(3-哌啶基丙氧基)苯基]- $5 \mathrm{H}$-狮并 [1,2-c]异 埕啉-5,11(6H)-二酮 $(\mathbf{1 b})$ 的合成

以化合物 $72.71 \mathrm{~g}(8.0 \mathrm{mmol})$ 和 $N$-(3-氯丙基)哌啶盐 酸盐 $4.73 \mathrm{~g}$ (24.0 mmol)为原料, 采用与 $1 \mathrm{a}$ 相同合成方 法，得深红色固体 $2.72 \mathrm{~g}$, 收率 $73 \%$. m.p. $132 \sim 138{ }^{\circ} \mathrm{C}$; ${ }^{1} \mathrm{H}$ NMR $\left(400 \mathrm{MHz}, \mathrm{CDCl}_{3}\right) \delta: 8.74(\mathrm{~d}, J=8.0 \mathrm{~Hz}, 1 \mathrm{H}$, $\operatorname{ArH}), 8.37$ (d, $J=8.0 \mathrm{~Hz}, 1 \mathrm{H}, \operatorname{ArH}), 7.78(\mathrm{t}, J=8.0 \mathrm{~Hz}$, $1 \mathrm{H}, \operatorname{ArH}), 7.56$ (d, $J=7.2 \mathrm{~Hz}, 1 \mathrm{H}, \mathrm{ArH}), 7.49$ (t, $J=7.2$ $\mathrm{Hz}, 1 \mathrm{H}, \mathrm{ArH}), 7.34$ (d, $J=8.8 \mathrm{~Hz}, 2 \mathrm{H}, \mathrm{ArH}), 7.26$ (t, $J=$ $7.6 \mathrm{~Hz}, 1 \mathrm{H}, \mathrm{ArH}), 7.14(\mathrm{~d}, J=8.8 \mathrm{~Hz}, 2 \mathrm{H}, \mathrm{ArH}), 7.07$ (t, $J=7.6 \mathrm{~Hz}, 1 \mathrm{H}, \mathrm{ArH}), 5.70$ (d, $J=7.6 \mathrm{~Hz}, 1 \mathrm{H}, \mathrm{ArH}), 4.17$ (t, $\left.J=7.6 \mathrm{~Hz}, 2 \mathrm{H}, \mathrm{OCH}_{2} \mathrm{CH}_{2} \mathrm{CH}_{2} \mathrm{~N}\right), 2.66(\mathrm{t}, J=7.6 \mathrm{~Hz}$, $2 \mathrm{H}, \mathrm{OCH}_{2} \mathrm{CH}_{2} \mathrm{CH}_{2} \mathrm{~N}$ ), 2.40 2.61 (m, 4H, 2,6-piperidyl$\mathrm{H}), 2.14 \sim 2.17\left(\mathrm{~m}, 2 \mathrm{H}, \mathrm{OCH}_{2} \mathrm{CH}_{2} \mathrm{CH}_{2} \mathrm{~N}\right), 1.69 \sim 1.74(\mathrm{~m}$, 4H, 3,5-piperidyl-H), 1.52 1.54 (m, 2H, 4-piperidyl-H); IR (KBr) v: 3463, 2965, 1606, 1502, 1410, 1251, 1074, $806,759,528 \mathrm{~cm}^{-1}$; MS (EI) $\mathrm{m} / \mathrm{z}: 464[\mathrm{M}]^{+}$.

3.6.3 6-[4-(2-二乙胺基乙氧基)苯基]-5H-狮并 [1,2-c] 异喹啉-5,11(6H)-二酮(1c)的合成

以化合物 $72.71 \mathrm{~g}(8.0 \mathrm{mmol})$ 和 $N$-(2-氯乙基)二乙胺 盐酸盐 $4.13 \mathrm{~g}(24.0 \mathrm{mmol})$ 为原料, 采用与 $1 \mathrm{a}$ 相同合成 方法, 得深红色固体 $2.41 \mathrm{~g}$, 收率 69\%. m.p. 127 $132{ }^{\circ} \mathrm{C} ;{ }^{1} \mathrm{H}$ NMR $\left(400 \mathrm{MHz}, \mathrm{CDCl}_{3}\right) \delta: 8.75(\mathrm{~d}, J=8.0$ 
$\mathrm{Hz}, 1 \mathrm{H}, \mathrm{ArH}), 8.35$ (d, $J=8.0 \mathrm{~Hz}, 1 \mathrm{H}, \mathrm{ArH}), 7.79$ (t, $J=$ $8.0 \mathrm{~Hz}, 1 \mathrm{H}, \mathrm{ArH}), 7.57$ (d, $J=7.2 \mathrm{~Hz}, 1 \mathrm{H}, \mathrm{ArH}), 7.50$ (t, $J=7.2 \mathrm{~Hz}, 1 \mathrm{H}, \mathrm{ArH}), 7.40$ (d, $J=8.8 \mathrm{~Hz}, 2 \mathrm{H}, \operatorname{ArH}), 7.26$ (t, $J=7.6 \mathrm{~Hz}, 1 \mathrm{H}, \mathrm{ArH}), 7.17(\mathrm{~d}, J=8.8 \mathrm{~Hz}, 2 \mathrm{H}, \mathrm{ArH}), 7.01$ (t, $J=7.6 \mathrm{~Hz}, 1 \mathrm{H}, \mathrm{ArH}), 5.70(\mathrm{~d}, J=7.2 \mathrm{~Hz}, 1 \mathrm{H}, \mathrm{ArH})$, $4.73\left(\mathrm{t}, J=5.7 \mathrm{~Hz}, 2 \mathrm{H}, \mathrm{OCH}_{2} \mathrm{CH}_{2} \mathrm{~N}\right), 3.57(\mathrm{t}, J=6.0 \mathrm{~Hz}$, $\left.2 \mathrm{H}, \mathrm{OCH}_{2} \mathrm{CH}_{2} \mathrm{~N}\right), 3.31 \sim 3.42\left(\mathrm{~m}, 4 \mathrm{H}, \mathrm{N}\left[\mathrm{CH}_{2} \mathrm{CH}_{3}\right)_{2}\right]$, $1.47 \sim 1.59\left[\mathrm{~m}, 6 \mathrm{H}, \mathrm{N}\left(\mathrm{CH}_{2} \mathrm{CH}_{3}\right)_{2}\right]$; IR $(\mathrm{KBr}) v: 3463,2965$, 1668, 1606, 1502, 1410, 1251, 1074, 806, 759, $528 \mathrm{~cm}^{-1}$; MS (EI) $m / z: 438[\mathrm{M}]^{+}$.

3.6 .4 6-[4-(3-二乙胺基丙氧基)苯基]-5H-狮并 $[1,2-c]$ 异喹啉-5,11(6H)-二酮(1d) 的合成

以化合物 $72.71 \mathrm{~g}(8.0 \mathrm{mmol})$ 和 $N$-(3-氯丙基)二乙胺 盐酸盐 $4.46 \mathrm{~g}$ (24.0 mmol)为原料, 采用与 $1 \mathbf{a}$ 相同合成 方法, 得深红色固体 2.43 g, 收率 67\%. m.p. 122 $124{ }^{\circ} \mathrm{C} ;{ }^{1} \mathrm{H}$ NMR (400 MHz, $\left.\mathrm{CDCl}_{3}\right) \delta: 8.72$ (d, $J=8.0$ $\mathrm{Hz}, 1 \mathrm{H}, \mathrm{ArH}), 8.34$ (d, $J=8.0 \mathrm{~Hz}, 1 \mathrm{H}, \mathrm{ArH}), 7.77$ (t, $J=$ $8.0 \mathrm{~Hz}, 1 \mathrm{H}, \mathrm{ArH}), 7.56$ (d, J=7.2 Hz, 1H, ArH), 7.49 (t, $J=7.2 \mathrm{~Hz}, 1 \mathrm{H}, \mathrm{ArH}), 7.36$ (d, $J=8.4 \mathrm{~Hz}, 2 \mathrm{H}, \operatorname{ArH}), 7.26$ (t, $J=7.6 \mathrm{~Hz}, 1 \mathrm{H}, \mathrm{ArH}), 7.12(\mathrm{~d}, J=8.4 \mathrm{~Hz}, 2 \mathrm{H}, \mathrm{ArH})$, 7.07 (t, $J=7.6 \mathrm{~Hz}, 1 \mathrm{H}, \operatorname{ArH}), 5.67$ (d, $J=7.6 \mathrm{~Hz}, 1 \mathrm{H}$, $\mathrm{ArH}), 4.26\left(\mathrm{t}, J=4.8 \mathrm{~Hz}, 2 \mathrm{H}, \mathrm{OCH}_{2} \mathrm{CH}_{2} \mathrm{CH}_{2} \mathrm{~N}\right), 3.32$ (t, $\left.J=7.6 \mathrm{~Hz}, 2 \mathrm{H}, \mathrm{OCH}_{2} \mathrm{CH}_{2} \mathrm{CH}_{2} \mathrm{~N}\right), 3.21 \sim 3.25(\mathrm{~m}, 4 \mathrm{H}$, $\left.\mathrm{N}\left(\mathrm{CH}_{2} \mathrm{CH}_{3}\right)_{2}\right), \quad 2.49 \sim 2.50\left(\mathrm{~m}, 2 \mathrm{H}, \quad \mathrm{OCH}_{2} \mathrm{CH}_{2} \mathrm{CH}_{2} \mathrm{~N}\right)$, $1.48 \sim 1.52\left[\mathrm{~m}, 6 \mathrm{H}, \mathrm{N}\left(\mathrm{CH}_{2} \mathrm{CH}_{3}\right)_{2}\right]$; IR (KBr) v: 3440, 2964, $1666,1607,1503,1413,1251,760,532 \mathrm{~cm}^{-1}$; MS (EI) $m / z: 452[\mathrm{M}]^{+}$.

3.6.5 6-[4-(2-(4-甲基哌嗪基)乙氧基)苯基]-5H-狮并 [1,2-c]异喹啉-5,11(6H)-二酮(1e) 的合成

以化合物 $72.71 \mathrm{~g}$ (8.0 mmol)和 $N$-(2-氯乙基)-4-甲 基哌嗪二盐酸盐 $5.65 \mathrm{~g}(24.0 \mathrm{mmol})$ 为原料, 采用与 $\mathbf{1 a}$ 相同合成方法, 得深红色固体 $3.01 \mathrm{~g}$, 收率 81\%. m.p. $136 \sim 140{ }^{\circ} \mathrm{C} ;{ }^{1} \mathrm{H}$ NMR $\left(400 \mathrm{MHz}, \mathrm{CDCl}_{3}\right) \delta: 8.74(\mathrm{~d}, J=$ $7.6 \mathrm{~Hz}, 1 \mathrm{H}, \mathrm{ArH}), 8.35$ (d, $J=7.6 \mathrm{~Hz}, 1 \mathrm{H}, \mathrm{ArH}), 7.79$ (t, $J=8.0 \mathrm{~Hz}, 1 \mathrm{H}, \operatorname{ArH}), 7.57$ (d, $J=6.8 \mathrm{~Hz}, 1 \mathrm{H}, \operatorname{ArH}), 7.50$ (t, $J=7.6 \mathrm{~Hz}, 1 \mathrm{H}, \mathrm{ArH}), 7.36$ (d, $J=8.8 \mathrm{~Hz}, 2 \mathrm{H}, \mathrm{ArH})$, $7.26(\mathrm{t}, J=7.6 \mathrm{~Hz}, 1 \mathrm{H}, \operatorname{ArH}), 7.14$ (d, $J=8.8 \mathrm{~Hz}, 2 \mathrm{H}$, $\operatorname{ArH}), 7.08$ (t, $J=7.2 \mathrm{~Hz}, 1 \mathrm{H}, \operatorname{ArH}), 5.70(\mathrm{~d}, J=7.2 \mathrm{~Hz}$, $1 \mathrm{H}, \mathrm{ArH}), 4.28\left(\mathrm{t}, J=5.4 \mathrm{~Hz}, 2 \mathrm{H}, \mathrm{OCH}_{2} \mathrm{CH}_{2} \mathrm{~N}\right), 3.42$ (t, $\left.J=5.4 \mathrm{~Hz}, 2 \mathrm{H}, \quad \mathrm{OCH}_{2} \mathrm{CH}_{2} \mathrm{~N}\right), 3.06 \sim 3.19(\mathrm{~m}, 8 \mathrm{H}$, piperazinyl-H), $2.81\left(\mathrm{~s}, 3 \mathrm{H}, \mathrm{NCH}_{3}\right)$; IR (KBr) v: 3545, 3447, 2926, 1669, 1608, 1503, 1411, 1248, 760, $531 \mathrm{~cm}^{-1}$; MS (EI) $m / z: 465[\mathrm{M}]^{+}$.
3.6.6 6-[4-(3-(4-甲基哌嗪基)丙氧基)苯基]-5 $\mathrm{H}$-狮并 [1,2-c]异喹啉-5,11(6H)-二酮(1f) 的合成

以化合物 $72.71 \mathrm{~g}(8.0 \mathrm{mmol})$ 和 $N$-(3-氯丙基)-4-甲 基哌嗪二盐酸盐 $6.22 \mathrm{~g}(24.0 \mathrm{mmol})$ 为原料, 采用与 $\mathbf{1 a}$ 相同合成方法, 得深红色固体 $2.20 \mathrm{~g}$, 收率 57\%. m.p. $179 \sim 182{ }^{\circ} \mathrm{C} ;{ }^{1} \mathrm{H}$ NMR (400 MHz, $\left.\mathrm{CDCl}_{3}\right) \delta: 8.72(\mathrm{~d}, J=$ $8.0 \mathrm{~Hz}, 1 \mathrm{H}, \mathrm{ArH}), 8.35$ (d, $J=8.0 \mathrm{~Hz}, 1 \mathrm{H}, \operatorname{ArH}), 7.76$ (t, $J=8.0 \mathrm{~Hz}, 1 \mathrm{H}, \mathrm{ArH}), 7.55$ (d, $J=7.2 \mathrm{~Hz}, 1 \mathrm{H}, \mathrm{ArH}), 7.48$ (t, $J=7.6 \mathrm{~Hz}, 1 \mathrm{H}, \mathrm{ArH}), 7.34$ (d, $J=8.8 \mathrm{~Hz}, 2 \mathrm{H}, \mathrm{ArH})$, $7.25(\mathrm{t}, J=7.6 \mathrm{~Hz}, 1 \mathrm{H}, \operatorname{ArH}), 7.13(\mathrm{~d}, J=8.8 \mathrm{~Hz}, 2 \mathrm{H}$, ArH), 7.06 (t, $J=7.2 \mathrm{~Hz}, 1 \mathrm{H}, \operatorname{ArH}), 5.68$ (d, $J=7.6 \mathrm{~Hz}$, $1 \mathrm{H}, \mathrm{ArH}), 4.16\left(\mathrm{t}, J=6.4 \mathrm{~Hz}, 2 \mathrm{H}, \mathrm{OCH}_{2} \mathrm{CH}_{2} \mathrm{CH}_{2} \mathrm{~N}\right)$, $2.61 \sim 2.65\left(\mathrm{~m}, 2 \mathrm{H}, \mathrm{OCH}_{2} \mathrm{CH}_{2} \mathrm{CH}_{2} \mathrm{~N}\right), 2.53 \sim 2.62(\mathrm{~m}, 8 \mathrm{H}$, piperazinyl-H), $2.36\left(\mathrm{~s}, 3 \mathrm{H}, \mathrm{NCH}_{3}\right), 2.06 \sim 2.09(\mathrm{~m}, 2 \mathrm{H}$, $\mathrm{OCH}_{2} \mathrm{CH}_{2} \mathrm{CH}_{2} \mathrm{~N}$ ); IR (KBr) v: 3470, 2926, 2792, 1670, 1608, 1504, 1414, 1251, 762, $529 \mathrm{~cm}^{-1}$; MS (EI) $\mathrm{m} / z: 479$ $[\mathrm{M}]^{+}$.

3.6.7 6-[4-(2-二甲胺基乙氧基)苯基]-5H-狮并[1,2-c] 异喹啉-5,11(6H)-二酮(1g)的合成

以化合物 $72.71 \mathrm{~g}(8.0 \mathrm{mmol})$ 和 $N$-(2-氯乙基)二甲胺 盐酸盐 $3.46 \mathrm{~g}$ (24.0 mmol)为原料, 采用与 $1 \mathrm{a}$ 相同合成 方法, 得深红色固体 $2.41 \mathrm{~g}$, 收率 74\%. m.p. 152 $156{ }^{\circ} \mathrm{C} ;{ }^{1} \mathrm{H}$ NMR (400 MHz, $\left.\mathrm{CDCl}_{3}\right) \delta: 8.73$ (d, $J=8.0$ $\mathrm{Hz}, 1 \mathrm{H}, \mathrm{ArH}), 8.36$ (d, $J=8.0 \mathrm{~Hz}, 1 \mathrm{H}, \mathrm{ArH}), 7.78$ (t, $J=$ $7.6 \mathrm{~Hz}, 1 \mathrm{H}, \mathrm{ArH}), 7.56$ (d, $J=7.2 \mathrm{~Hz}, 1 \mathrm{H}, \mathrm{ArH}), 7.50$ (t, $J$ $=7.6 \mathrm{~Hz}, 1 \mathrm{H}, \operatorname{ArH}), 7.35(\mathrm{~d}, J=8.8 \mathrm{~Hz}, 2 \mathrm{H}, \operatorname{ArH}), 7.26(\mathrm{t}$, $J=7.6 \mathrm{~Hz}, 1 \mathrm{H}, \mathrm{ArH}), 7.17(\mathrm{~d}, J=8.8 \mathrm{~Hz}, 2 \mathrm{H}, \mathrm{ArH}), 7.05$ (t, $J=7.2 \mathrm{~Hz}, 1 \mathrm{H}, \mathrm{ArH}), 5.67$ (d, $J=7.6 \mathrm{~Hz}, 1 \mathrm{H}, \mathrm{ArH}), 4.22$ $\left(\mathrm{t}, J=5.6 \mathrm{~Hz}, 2 \mathrm{H}, \mathrm{OCH}_{2} \mathrm{CH}_{2} \mathrm{~N}\right), 2.86(\mathrm{t}, J=5.6 \mathrm{~Hz}, 2 \mathrm{H}$, $\left.\mathrm{OCH}_{2} \mathrm{CH}_{2} \mathrm{~N}\right), 2.43$ [s, 6H, N($\left.\left(\mathrm{CH}_{3}\right)_{2}\right]$; IR ( $\left.\mathrm{KBr}\right) v: 3583$, 3442, 2935, 1668, 1607, 1502, 1411, 1249, 762, $532 \mathrm{~cm}^{-1}$; MS (EI) $m / z: 410[\mathrm{M}]^{+}$.

3.6.8 6-[4-(3-二甲胺基丙氧基)苯基]-5 $H$-狮并 $[1,2-c]$ 异喹啉-5,11(6H)-二酮(1h) 的合成

以化合物 $72.71 \mathrm{~g}(8.0 \mathrm{mmol})$ 和 $N$-(3-氯丙基)哌啶盐 酸盐 $3.79 \mathrm{~g}(24.0 \mathrm{mmol})$ 为原料, 采用与 $1 \mathbf{a}$ 相同合成方 法, 得深红色固体 $1.98 \mathrm{~g}$, 收率 58\%. m.p. 148 152 ${ }^{\circ} \mathrm{C}$; ${ }^{1} \mathrm{H}$ NMR (400 MHz, $\left.\mathrm{CDCl}_{3}\right) \delta: 8.73(\mathrm{~d}, J=8.0 \mathrm{~Hz}, 1 \mathrm{H}$, ArH), 8.36 (d, $J=8.0 \mathrm{~Hz}, 1 \mathrm{H}, \operatorname{ArH}), 7.78$ (t, $J=7.6 \mathrm{~Hz}$, $1 \mathrm{H}, \mathrm{ArH}), 7.56$ (d, $J=7.2 \mathrm{~Hz}, 1 \mathrm{H}, \mathrm{ArH}), 7.49$ (t, $J=7.6$ $\mathrm{Hz}, 1 \mathrm{H}, \mathrm{ArH}), 7.34$ (d, $J=8.8 \mathrm{~Hz}, 2 \mathrm{H}, \operatorname{ArH}), 7.26$ (t, $J=$ $7.2 \mathrm{~Hz}, 1 \mathrm{H}, \mathrm{ArH}), 7.14$ (d, J=8.8 Hz, 2H, ArH), 7.09 (t, $J=7.2 \mathrm{~Hz}, 1 \mathrm{H}, \mathrm{ArH}), 5.69$ (d, $J=7.2 \mathrm{~Hz}, 1 \mathrm{H}, \mathrm{ArH}), 4.17$ (t, $\left.J=6.4 \mathrm{~Hz}, 2 \mathrm{H}, \mathrm{OCH}_{2} \mathrm{CH}_{2} \mathrm{CH}_{2} \mathrm{~N}\right), 2.55 \sim 2.58(\mathrm{~m}, 2 \mathrm{H}$, $\left.\mathrm{OCH}_{2} \mathrm{CH}_{2} \mathrm{CH}_{2} \mathrm{~N}\right), 2.34\left[\mathrm{~s}, 6 \mathrm{H}, \mathrm{N}\left(\mathrm{CH}_{3}\right)_{2}\right], 2.06 \sim 2.09(\mathrm{~m}$, 
$\left.2 \mathrm{H}, \mathrm{OCH}_{2} \mathrm{CH}_{2} \mathrm{CH}_{2} \mathrm{~N}\right)$; IR (KBr) v: 3478, 3436, 2950, $1668,1607,1504,1412,1249,760,532 \mathrm{~cm}^{-1}$; MS (EI) $m / z: 424[\mathrm{M}]^{+}$.

辅助材料(Supporting Information) 中间体 $c i s-\mathbf{2 a} \sim \mathbf{2 c}$ 的 ESI-MS 和 ${ }^{1} \mathrm{H}$ NMR 图谱, 目标化合物 $\mathbf{1 a} \sim \mathbf{1 h}$ 的 EI-MS、IR 和 ${ }^{1} \mathrm{H}$ NMR 图谱. 这些材料可以免费从本刊 网站(http://sioc-journal.cn/)上下载.

\section{References}

[1] Conda-Sheridan, M.; Reddy, P. V. N.; Morrell, A.; Cobb, B. T.; Marchand, C.; Agama, K.; Chergui, A.; Renaud, A.; Stephen, A. G.; Bindu, L. K.; Pommier, Y.; Cushman, M. J. Med. Chem. 2013, $56,182$.

[2] (a) Morrell, A.; Placzek, M.; ParmLey, S.; Antony, S.; Dexheimer, T. S.; Pommier, Y.; Cushman, M. J. Med. Chem. 2007, 50, 4419.

(b) Cushman, M.; Jayaraman, M.; Vroman, J. A.; Fukunaga, A. K.; Fox, B. M.; Kohlhagen, G.; Strumberg, D.; Pommier, Y. J. Med. Chem. 2000, 43, 3688

(c) Zhang, X. Y.; Wang, R. B.; Zhao, L.; Lu, N.; Wang, J. B.; You, Q. D.; Li, Z. Y.; Guo, Q. L. Bioorg. Med. Chem. Lett. 2012, 22, 1276.

[3] Wang, T. L.; Xiang, H.; Xu, X. L.; Xiao, H.; You, Q. D. Chin. J. Med. Chem. 2012, 22, 268 (in Chinese).

(王天麟, 向华, 徐晓丽, 肖红, 尤启冬, 中国药物化学杂志, 2012, 22, 268.)

[4] Wang, L. J.; Zeng, Y. Y.; Wang, T. L.; Liu, H. Y.; Xiao, H.; Xiang, H. PLoS One 2014, 9, e86509.
[5] Azizian, J.; Mohammadi, A. A.; Karimi, A. R.; Mohammadizadeh, M. R. J. Org. Chem. 2005, 70, 350.

[6] (a) Johnson, J. V.; Rauckman, S.; Baccanari, P. D.; Roth, B. J. Med. Chem. 1989, 32, 1942.

(b) Stanoeva, E.; Haimova, M. Khim. Geterotsikl. Soedin. 1984, 1587.

(c) Yamada, N.; Kadowaki, S.; Takahashi, K.; Umeu, K. Biochem. Pharmacol. 1992, 44, 1211.

[7] Cushman, M.; Cheng, L. J. Org. Chem, 1978, 19, 3781.

[8] (a) Xiao, X. S.; Morrell, A.; Fanwick, P. E.; Mark Cushmana, M. Tetrahedron 2006, 62, 9705.

(b) Cushman, M.; Gentry, J.; Dekow, F. W. J. Org. Chem. 1977, $42,1111$.

(c) González-López, M.; Shaw, J. T. Chem. Rev. 2009, 109, 164.

[9] Cushman, M.; Madaj, E. J. J. Org. Chem. 1987, 52, 907.

[10] (a) Azizian, J.; Mohammadi, A. A.; Soleimani, E.; Karimi, A. R.; Mohammadizadeh, M. R. J. Heterocyclic Chem. 2006, 43, 187.

(b) Wang, L. M.; Liu, J. J.; Tian, H,; Qian, C. T.; Sun, J. $A d v$. Synth. Catal. 2005, 347, 689.

(c) Yadav, J. S.; Subba, B. V. R.; Ramesh, A. R.; Narsaiah, A. V. Synthesis 2007, 20, 3191.

[11] Yu, N. F.; Bourel, L.; Deprez, B.; Gesquiere, J. C. Tetrahedron Lett. 1998, 39, 829

[12] Vara, Y.; Bello, T.; Aldaba, E.; Arrieta, A.; Pizarro, J. L.; Arriortua, M.; Lopez, X.; Cossío, F. P. Org. Lett. 2008, 21, 4759.

[13] Tong, G. J.; Fan, W.; Jiang, B. Chin. J. Org. Chem. 2013, 33, 2578 (in Chinese).

(倐光进, 范威, 姜波, 有机化学, 2013, 33, 2578.)

[14] Zen, C.; Qu, Z. Q. Chin. J. Org. Chem. 2013, 33, 2533 (in Chinese).

(曾琛，曲占庆，有机化学, 2013, 33, 2533.) 